\title{
Silicon Photonics for Optical Interconnects
}

\author{
${ }^{1}$ Frederic Gardes, ${ }^{2}$ Kapil Debnath and ${ }^{2}$ Liam O'Faolain Member, IEEE \\ ${ }^{1}$ Optoelectronics Research Centre, University of Southampton, Southampton SO17 1BJ, United Kingdom \\ ${ }^{2}$ SUPA, School of Physics and Astronomy, University of St Andrews, North Haugh, St Andrews, Fife, KY16 9SS, \\ Scotland, UK \\ e-mail:F.Gardes@soton.ac.uk
}

\begin{abstract}
Integration density, channel scalability, low switching energy and low insertion loss are the major prerequisites for on-chip Wavelength Division Multiplexing systems. A number of device geometries have already been demonstrated that fulfil these criteria, at least in part, but combining all of the requirements is still a difficult challenge. Photonic crystal cavities provide the ultimate confinement of light in space and time, giving the potential to realise devices with the lowest energy consumption and highest integration densities. Here, we demonstrate Photonic Crystal cavity modulators with very low switching energies and high speed Mach Zehnder interferometer modulators and discuss their relative merits.
\end{abstract}

Keywords: optical modulators, photonic crystals, silicon photonics, photodetectors.

\section{INTRODUCTION}

Integration density, channel scalability, low switching energy, high speed operation and low insertion loss are the major prerequisites for on-chip Wavelength Division Multiplexing (WDM) systems. A number of device geometries have already been demonstrated that fulfill these criteria, at least in part, but combining all of the requirements is still a difficult challenge.

Here we describe two families of optical modulators for datacommunications, high speed Mach Zehnder Interferometers and compact Photonic crystals cavities with low switching energies and discuss their respective advantages. Both use the free carrier dispersions effect which is the most popular means to achieve high-speed optical modulation in silicon [1]. A change in free carrier density results in a change in the refractive index of the material, which modifies the resonance wavelength of an optical filter- the PhC cavity approach - or optical phase of light passing through it- the MZI approach. The modulation speed is determined by how fast the free carriers can be injected into or removed from the volume of modulator and the switching by the number of carriers that must be injected to realise the desired effect.

\section{The Mach Zehnder Interferometer approach}

Modulation based on the depletion of carriers in a pn junction is not limited by the minority carrier recombination lifetime giving rise to very fast response time. In 2007, Liu et al. [2] experimentally demonstrated one of the first $p n$ carrier depletion-based silicon optical modulators. Since, the research trend has been to move towards smaller waveguides to reduce power consumption and device real estate leading to structures such as the one recently reported by Gardes et al. [3] in 2009. One of the principal issues with the fabrication of pn junction in a rib waveguide was the inaccuracy of the positioning of the junction due to processing variations. This issue was solved in [4] with a new design of carrier depletion based silicon optical modulator in which a self aligned process was used to form the $p n$ junction, shown in figure 1.

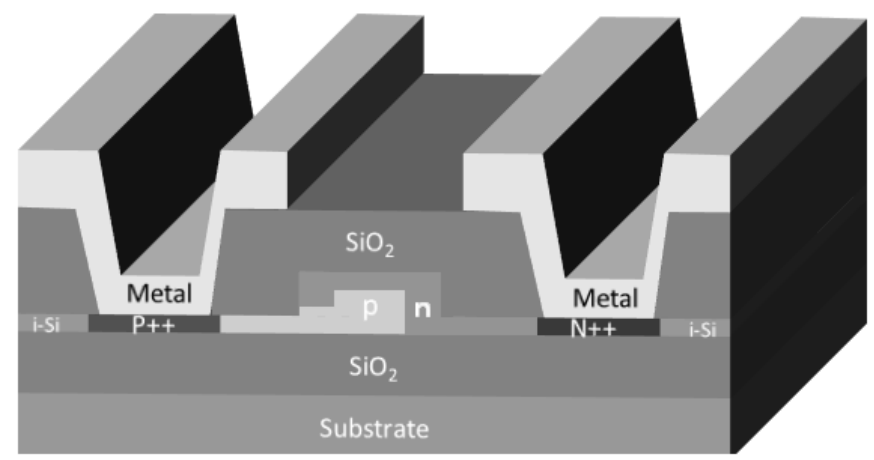

Figure 1 - Cross sectional diagram of self-aligned pn junction phase modulator

The phase modulators were formed by implanting a $p n$ junction in a rib waveguides, the dimensions of which were $400 \mathrm{~nm}$ in height, $410 \mathrm{~nm}$ in width and $100 \mathrm{~nm}$ in slab height. The implantation is carried out such that the 
rib waveguide is surrounded by an $\mathrm{n}$ type doping region and the centre of the waveguide is formed by a p region. Highly doped regions are formed below the contacts to ensure that resistive contacts are realised. These are positioned sufficiently far away from the waveguide edge such that excessive optical loss is avoided. The phase modulators are incorporated into Mach Zehnder interferometers (MZI) that translate the phase modulation into intensity modulation. Phase modulators are placed into both arms of the MZI to balance the optical power and therefore permit a large static extinction ratio. Coplanar waveguide (CPW) electrodes are used to drive the modulator at high frequency and were designed to have a characteristic impedance of $50 \mathrm{ohms}$.
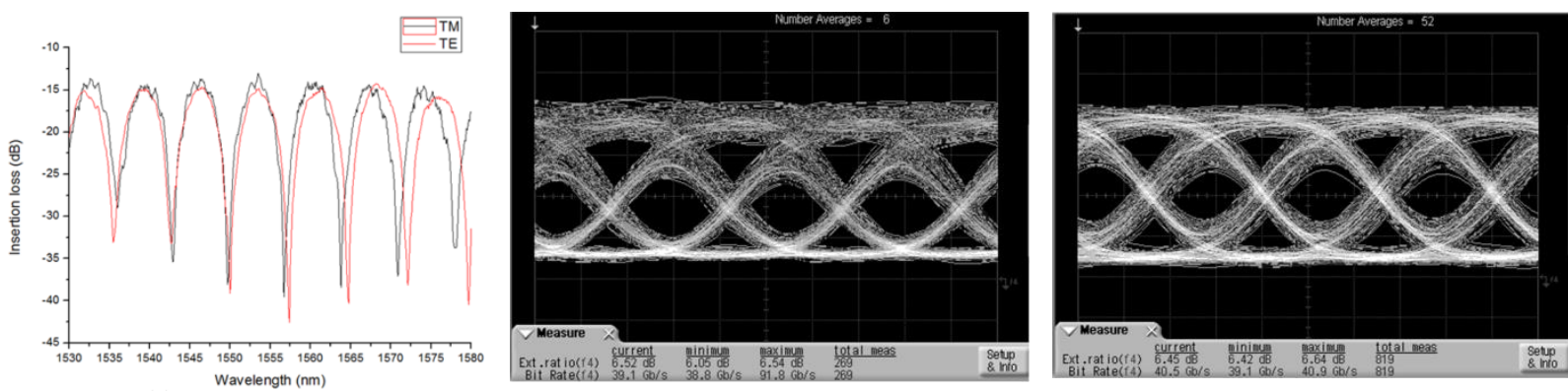

Figure 2 - (Left to right) Typical transmission specturm; Eye diagram for a 1350 micrometer long device measured at $40 \mathrm{~Gb} / \mathrm{s}$, and at a wavelength of $\sim 1557 \mathrm{~nm}$ for TE polarisation. The extinction ratio is approximately $6.5 \mathrm{~dB}$; Eye diagram for a 1350 micrometers long device measured at 40Gb/s, and at a wavelength of $\sim 1557 \mathrm{~nm}$ for TM polarisation. The extinction ratio is approximately $6.5 \mathrm{~dB}$.

The device fabrication was carried out in the clean room facilities at CEA-LETI using Silicon On Insulator with a $400 \mathrm{~nm}$ device layer SOI with a $1 \mu \mathrm{m}$ thick buried oxide layer. The figure of merit for the efficiency, V $\pi$.L $\pi$ (voltage-length required to achieve a $\pi$ phase shift for a given length), was extracted from the transmission shift against reverse voltage for TE and TM polarisations and found to be $\sim 11 \mathrm{~V} . \mathrm{cm}$ and $\sim 14 \mathrm{~V} . \mathrm{cm}$. The on-chip insertion loss is $\sim 15 \mathrm{~dB}$ (includes $\sim 5.7 \mathrm{~dB}$ and $6.7 \mathrm{~dB}$ of passive waveguide transmission loss for TE and TM polarisations respectively). $5.7 \mathrm{~dB} / \mathrm{mm}$ loss was introduced by the doping in the waveguides for the TE polarization and is responsible for the majority of the phase shifter insertion loss.

A 40Gb/s PRBS generator was used for the data rate measurements. The measurements were performed at $1557 \mathrm{~nm}$ for both polarisations with a data stream of $40 \mathrm{~Gb} / \mathrm{s}$ for TM and TE polarisations as shown in figure 3 . Similar extinction ratios achieved for both TM and TE polarisations, approximately $6.5 \mathrm{~dB}$. At a data rate of $40 \mathrm{~Gb} / \mathrm{s}$ and with an input swing voltage is $6 \mathrm{~V}$, the power consumption is estimated to be about $4.5 \mathrm{pJ} / \mathrm{bit}$.

\section{Photonic Crystal Cavities}

The building block of our photonic crystal modulator is an all-pass filter comprising of a photonic crystal cavity vertically coupled to a bus waveguide [5]. In order to efficiently couple light ebetween two optical modes, two conditions must be satisfied: a spatial overlap must exist between the evanescent parts of the modes and they have to have matching k-vectors. The first condition is attained by optimizing the physical overlap between the two modes. The small mode volume of the photonic crystal cavity then helps to fulfill the second condition. Due to the smaller mode volume, the corresponding Fourier space distribution or k-space distribution expands, provide a rich distribution of $\mathrm{k}$-vectors that may match that of the bus waveguide. 
a)

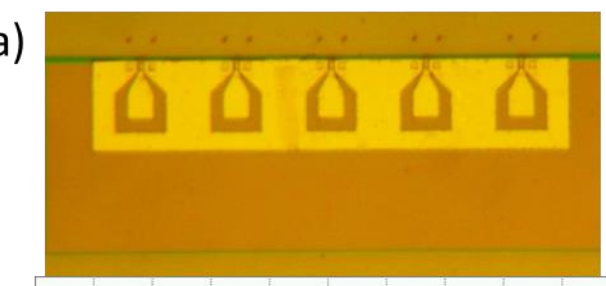

C)

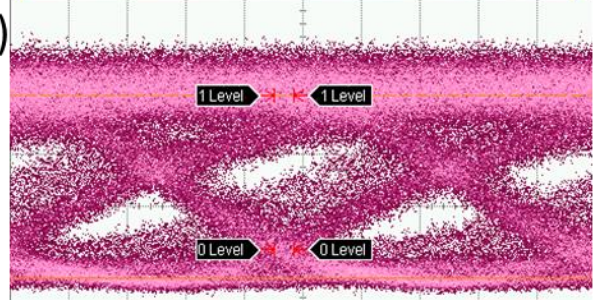

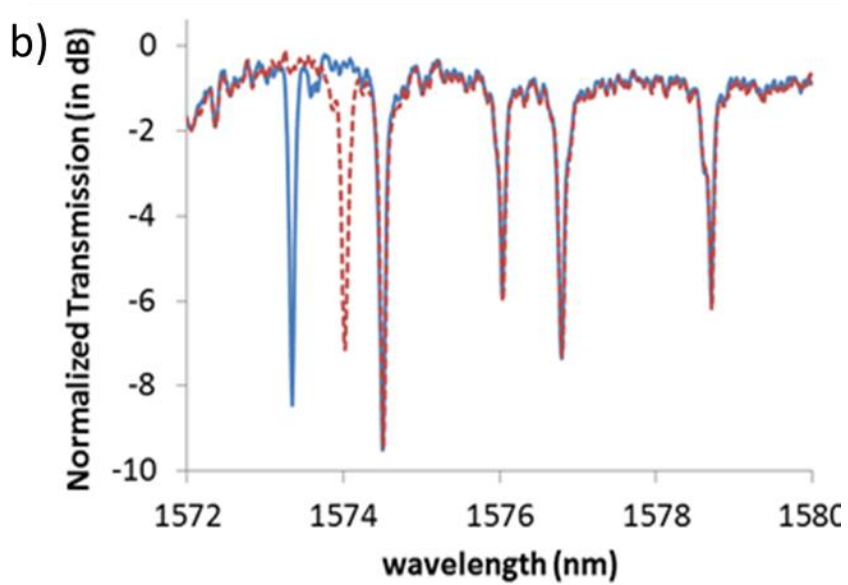

Figure 3:a) 5 cascaded photonic crystal modulators b) the corresponding transmission spectrum (blue). When a voltage is applied to one cavity the corresponding resonance shifts (red), c) The corresponding eye diagram

With a suitably designed $\mathrm{PhC}$ cavity, matching may be achieved to a large mode area, low refractive index contrast waveguide. This is a particularly powerful means of coupling a low index waveguide to a photonic crystal resonator, allowing very high efficiencies be attained, while creating the minimum perturbation to the resonator mode. Additionally, as the resonator is naturally wavelength selective, the channel may be modulated while in the bus, avoiding the need for complex multiplexors/demultiplexors. Thus, everything required to modulate an individual channel may be contained in an area of approximately $20 \mathrm{um} \times 20 \mathrm{um}$. As large arrays of single mode $\mathrm{PhC}$ cavities can be made, this system is very scalable and has potential for $1 \mathrm{Tbit} / \mathrm{s}$ data transmission [6]. At resonance, the extinction ratio is almost $7 \mathrm{~dB}$ while the overall quality factor is maintained at 50000 .

Modulation and multiplexing is achieved by cascading five $\mathrm{PhC}$ cavities with slightly different resonance wavelength, see figure $3 \mathrm{~b}$. Fine wavelength control is achieved by tuning the position of the four inner most holes around the cavity. The $\mathrm{PhC}$ cavities are embedded in $\mathrm{p}-\mathrm{i}-\mathrm{n}$ junctions in order to realise modulation. Here carrier injection is used to produce the high speed change in refractive index. The etching of the holes has a minimal effect on the electrical properties of the device [7]. The resonance wavelength of each cavity may be controlled independently. Thus, this technique allows us to modulate and multiplex simultaneously number of channels without the use of complex components, such as an Arrayed Waveguide Grating (AWG). Figure 3c shows modulation of this device at a data rate of $1.0 \mathrm{gbps}$. The maximum modulation speed was $1 \mathrm{Gbps}$ as carrier injection rather than depletion was used in this particular device. Further optimization will increase the electrical efficiency of the device. The ultimate limit on the modulation speed will be given by the photon lifetime in the cavity. For a Q-factor of 10,000, this limit is well in excess of $25 \mathrm{Gbit} / \mathrm{s}$. It is important to note that the required driving voltage for a $3 \mathrm{~dB}$ extinction ratio was only $0.7 \mathrm{~V}$, consequently the switching energy (Off-On state) was only $1.6 \mathrm{fJ}$, one of the lowest yet reported for a silicon electro-optic modulator.

\section{SUMMARY}

We have discussed some of the advantages of two very different types of silicon based optical modulator, the rib waveguide based MZI and the photonic crystal cavity. The MZI approach provides extremely high modulation speeds, whereas the PhC cavity approach provides the highest bandwidth densities and the lowest switching energies. The two approaches cover a very wide range of applications in optical interconnects.

\section{ACKNOWLEDGEMENTS}

This work was funded by the EPSRC under the UK Silicon Photonics project and through the Nanoscience ERA project LECSIN. Liam O'Faolain also acknowledges support from an SU2P fellowship (www.SU2P.com) and from a European Research Council Starting Grant (number 337508).

\section{REFERENCES}

[1] L. Liao, A. Liu, D. Rubin, J. Basak, Y. Chetrit, H. Nguyen, R. Cohen, N. Izhaky \& M. Paniccia "40Gbit/s silicon optical modulator for high-speed applications" Electronic Letters 43, 1196-1197 (2007)

[2] A. Liu, "High-speed optical modulation based on carrier depletion in a silicon waveguide," Opt. Express, vol. 15, pp. 660-668, 2007.

[3] F. Y. Gardes, A. Brimont, P. Sanchis, G. Rasigade, D. Marris-Morini, L. O'Faolain, F. Dong, J. M. Fedeli, P. Dumon, L. Vivien, T. F. Krauss, G. T. Reed, and J. Martí, "High-speed modulation of a compact silicon ring resonator based on a reverse-biased pn diode," Opt. Express, vol. 17, pp. 21986-21991, 2009 
[4] F. Y. Gardes, D. J. Thomson, N. G. Emerson, and G. T. Reed, "40 Gb/s silicon photonics modulator for TE and TM polarisations," Opt. Express, vol. 19, pp. 11804-11814.

[5] K. Debnath, K. Welna, M. Ferrera, K. Deasy, D. G. Lidzey \& L. O'Faolain "Highly efficient optical filter based on vertically coupled photonic crystal cavity and bus waveguide" Optics Letters 38, 154-156 (2013)

[6] K. Debnath, L. O'Faolain, F. Y. Gardes, A. G. Steffan, G. T. Reed \& T. F. Krauss "Cascaded modulator architecture for WDM applications" Optics Express 20, 27420-27428 (2012)

[7] P. Cardile, G. Franzò, R. L. Savio, M. Galli, T. F. Krauss, F. Priolo \& L. O. Faolain "Electrical conduction and optical properties of doped silicon-on-insulator photonic crystals" Applied Physics Letters 98, 203506 (2011)

[8] K. Debnath, F. Y. Gardes, A. P. Knights, G. T. Reed, T. F. Krauss \& L. O’Faolain "Dielectric waveguide vertically coupled to all-silicon photodiodes operating at telecommunication wavelengths" Applied Physics Letters 102, 171106 (2013) 